\title{
Multiobjective optimization applied to structural sizing of low cost university-class microsatellite projects
}

\author{
Ali Ravanbakhsh , Sebastián Franchini
}

\begin{abstract}
A B S T R A C T
In recent years, there has been continuing interest in the participation of university research groups in space technology studies by means of their own microsatellites. The involvement in such projects has some inherent challenges, such as limited budget and facilities. Also, due to the fact that the main objective of these projects is for educational purposes, usually there are uncertainties regarding their in orbit mission and scientific payloads at the early phases of the project. On the other hand, there are predetermined limitations for their mass and volume budgets owing to the fact that most of them are launched as an auxiliary payload in which the launch cost is reduced considerably. The satellite structure subsystem is the one which is most affected by the launcher constraints. This can affect different aspects, including dimensions, strength and frequency requirements. In this paper, the main focus is on developing a structural design sizing tool containing not only the primary structures properties as variables but also the system level variables such as payload mass budget and satellite total mass and dimensions. This approach enables the design team to obtain better insight into the design in an extended design envelope. The structural design sizing tool is based on analytical structural design formulas and appropriate assumptions including both static and dynamic models of the satellite. Finally, a Genetic Algorithm (GA) multiobjective optimization is applied to the design space. The result is a Pareto-optimal based on two objectives, minimum satellite total mass and maximum payload mass budget, which gives a useful insight to the design team at the early phases of the design.
\end{abstract}

\section{Introduction}

The ongoing interest of universities in microsatellite projects is a testament to their success in delivering results [1]. From 1981, the launch date of the first university-class microsatellite, named UoSAT-1, developed by University of Surrey [2], until 2005, more than 60 satellite projects have been conducted at universities [3], and more than $70 \%$ of these projects are included in the microsatellite class whose total mass is between $10-100 \mathrm{~kg}$. Also, based on the experiences of university-class microsatellite projects in different countries, it can be concluded that with these projects most of the basic and necessary infrastructures for space research and technology programs are obtained [1,4-8].

This work is done in the context of the UPMSat-2 project, which is a microsatellite under design and development at the Instituto Universitario de Microgravedad "Ignacio Da Riva" (IDR/UPM) of the Universidad Politécnica de Madrid. This project is a part of the IDR/UPM space research activities and is based on the previous experience of the UPMSat-1 microsatellite project launched on July 7, 1995 by Ariane IV [8-10]. University-class microsatellite projects 


\begin{tabular}{|c|c|c|c|}
\hline \multicolumn{2}{|c|}{ Nomenclature } & \multirow{3}{*}{$\begin{array}{l}k_{\varphi} \\
k_{j}\end{array}$} & \multirow{3}{*}{$\begin{array}{l}\text { equivalent rotational stiffness of launcher } \\
\text { adapter } \\
\text { equivalent lateral stiffness of the satellite } \\
\text { between each two trays, } j=5,6,7\end{array}$} \\
\hline Symbol & definition & & \\
\hline$A_{b}$ & equal leg angles L-bar cross section area & & \\
\hline$A_{S p}$ & side panel cross section area & $l_{b}$ & length of equal leg angles L-bar between each \\
\hline$A_{\text {sat }}$ & satellite model cross section area & & two trays \\
\hline$d_{b}$ & leg length of equal leg angles L-bar & $l_{s p}$ & length of side panel between each two trays \\
\hline$f_{\text {long/late }}$ & $\begin{array}{l}\text { longitudinal and lateral frequencies obtained } \\
\text { from structure sizing tool }\end{array}$ & $\begin{array}{l}l_{X Y} \\
L^{\prime}\end{array}$ & $\begin{array}{l}\text { satellite dimension in } X Y \text { direction } \\
\text { effective length for equal leg angles L-bar }\end{array}$ \\
\hline$F_{\text {long/late }}$ & longitudinal or lateral forces & $l_{Z}$ & satellite dimension in $Z$ direction \\
\hline & reduction factor & $m_{j}$ & concentrated mass on trays A, B, C \& D \\
\hline$f_{\text {r-long/late }}$ & $\begin{array}{l}\text { launcher required frequencies in longitudinal } \\
\text { and lateral directions } \\
\text { distance between two parallel side panels }\end{array}$ & $m_{c}$ & $\begin{array}{l}\text { satellite total mass calculated by structure } \\
\text { sizing tool }\end{array}$ \\
\hline $\begin{array}{l}I_{m} \\
I_{x}\end{array}$ & $\begin{array}{l}\text { satellite moment of inertia } \\
\text { satellite model second moment of area }\end{array}$ & $m_{i}$ & $\begin{array}{l}\text { initial mass introduced to structure } \\
\text { sizing tool }\end{array}$ \\
\hline$I_{x b}$ & $\begin{array}{l}\text { second moment of area for equal leg angles } \\
\text { L-bar }\end{array}$ & $\begin{array}{l}m_{\text {sat }} \\
M_{\max }\end{array}$ & $\begin{array}{l}\text { satellite total mass } \\
\text { maximum bending moment }\end{array}$ \\
\hline$k_{b j}$ & $\begin{array}{l}\text { equivalent lateral stiffness of equal leg angles } \\
\text { L-bar, } j=5,6,7\end{array}$ & $n_{\text {long/late }}$ & $\begin{array}{l}\text { longitudinal or lateral load factors (launcher } \\
\text { accelerations) }\end{array}$ \\
\hline$k_{\mathrm{c}}$ & buckling coefficient & $P_{c r}$ & critical buckling load \\
\hline$k_{s p j}$ & $\begin{array}{l}\text { equivalent lateral stiffness of side panel, } \\
j=5,6,7\end{array}$ & $q$ & $\begin{array}{l}\text { lateral uniform load distribution } \\
\text { satellite model first moment of area }\end{array}$ \\
\hline$k_{1}$ & $\begin{array}{l}\text { equivalent longitudinal stiffness of launcher } \\
\text { adapter }\end{array}$ & $\begin{array}{l}t_{s p} \\
t_{b}\end{array}$ & $\begin{array}{l}\text { side panel thickness } \\
\text { leg thickness of equal leg angles L-bar }\end{array}$ \\
\hline$k_{i}$ & $\begin{array}{l}\text { equivalent longitudinal stiffness of the satel- } \\
\text { lite between each two trays, } i=2,3,4\end{array}$ & $\begin{array}{l}\alpha \\
\beta\end{array}$ & $\begin{array}{l}\text { structure mass budget } \\
\text { payload mass budget }\end{array}$ \\
\hline
\end{tabular}

usually encounter challenges such as limited budget and facilities. To this end, applying simple design methods which can reduce the total cost of the project as well as its dependency on industrial facilities outside of the university is important. The opportunity of being launched as an auxiliary payload is one of the important strategies which effectively reduces the whole cost of these projects. On the other hand, this choice dictates some constraints to the design. These constraints are related to the allocation of limited mass and volume for the whole satellite, as well as specific strength and frequency requirements.

Besides fulfilling the abovementioned requirements, an optimized design considering single objectives or multiobjectives criteria is always required. The application of optimization techniques to aerospace design has a history of more than three decades. In [11] by SobieszczanskiSobieski, J. and Haftka, R.T., the evolution of multidisciplinary aerospace design optimization over the last few decades is reviewed. According to their findings, it can be concluded that there is much more progress in the optimization area of aircrafts compared to that of spacecraft design. This may be a result of complexity and interdisciplinary characteristics of spacecraft design. These characteristics have given rise to the fast-growing field of Multidisciplinary Design Optimization (MDO) methods. In [12] Mosher, T., has investigated selected multidisciplinary design optimization algorithms for conceptual spacecraft design. In his research, both classical and evolutionary optimization methods applicable to the spacecraft conceptual design problem are introduced. In [13] by Grooms, H.R. et al., an approach to spacecraft structural optimization considering system level considerations, such as the ease and availability of structure elements which not only affect the structural optimization, but the characteristics of the spacecraft as a whole, is presented.

By reviewing the works written in the field of structure and design optimization, it can be seen that structural design plays an important role in developing an optimum spacecraft design. Furthermore, the main subsystem which is affected by launcher constraints is the structure subsystem. Thus, having a comprehensive insight into the structural design is useful at the early phases of the design.

In this work, first a simple analytical model of the satellite structure subsystem has been established. Next, some specific design cases of the effects of different design variables on the design requirements are investigated. Finally, a multiobjective design optimization using Genetic Algorithm (GA) is applied to the developed structure sizing tool in order to obtain a Pareto-optimal in the design space. Using evolutionary algorithms such as GA have been applied in satellite design before [14-16]. In the present work, optimization objectives are selected as the minimization of satellite total mass and the maximization of payload mass budget. The first objective is always desired. The second one has been selected due to the uncertainties about the payload during early design phases in universityclass microsatellite projects. Also, having a maximum available mass budget for the payload will give versatility to the satellite platform for as yet undetermined payloads. The results of the optimization enables the design team to set optimum design baselines concerning both satellite 
system variables such as total mass and dimensions, and structure subsystem characteristics.

\section{Satellite configuration}

The satellite geometrical configuration is selected bearing in mind an easy manufacturing process which can be carried out by university facilities. Due to this approach, and based on other university microsatellite projects [4-7], the geometry is selected as a four-sided, square-based prism as shown in Fig. 1.

For subsystem equipment accommodation, four trays, A, B, C and D, including the bottom and top trays of the satellite are considered. The mass budgets of different subsystems including Attitude Determination and Control, Command and Data Handling, Power, Telecommunication, Thermal Control, Structure and Payload have been assumed based on design estimation relationships from a variety of sources [17-21] which are indicated in Table 1. In this study, the payload and structure mass budgets are not pre-assumed although their sum mass budgets should not exceed more than $55 \%$ of the satellite total mass. By reducing as much as possible the structure mass, there is more mass budget available for the payload. As mentioned in Section 1, this approach has special advantages for university-class microsatellite projects which in the early phases of the design have no exact information about the payload.

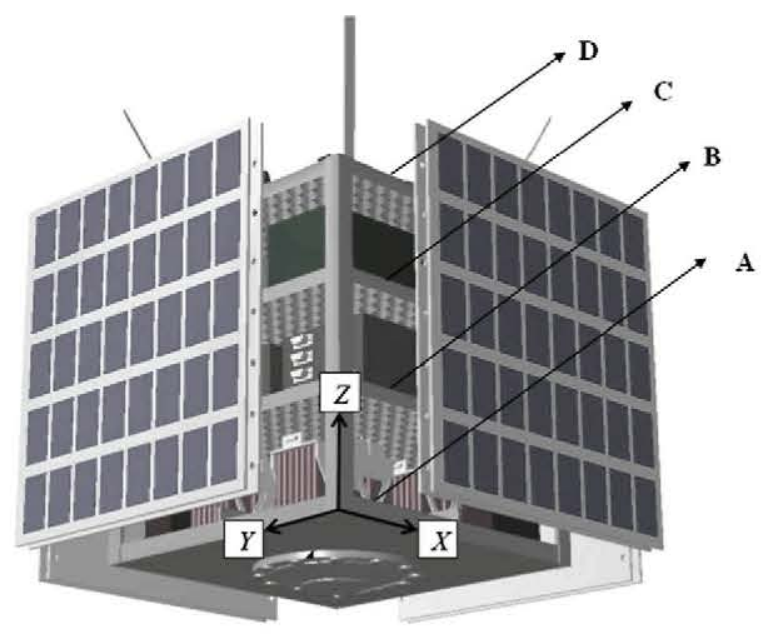

Fig. 1. Satellite geometrical configuration composed of trays A, B, C and D.

\section{Model description}

The structural model is combined of static and dynamic models. For static calculations, the analyses are done on each set of primary structures consisting of four equal leg angles L-bars as the satellite main frame, four plates as the side panels and four plates as the satellite trays. In order to facilitate low cost and low dependency on industry for structure manufacturing, the material is assumed to be an isotropic type space-qualified aluminum alloy. The trays are considered to be sized under an isogrid pattern to reduce the total mass. The satellite bottom tray is assumed to be clamped to the launcher, and maximum stress, maximum deflection and buckling of each primary structure element is analyzed.

For dynamic analysis, in order to estimate the natural frequencies, a simple mass-spring model is considered in both longitudinal and lateral directions. Structural modeling is done based on analytical design formulas from classic structure design references [22-25].

The structural calculations should meet some specific requirements of the foreseen satellite launcher. Usually, for university-class microsatellite projects, the exact information about the satellite launcher cannot be provided at the initial phases of the program. However, it is highly desirable to use the opportunity of being launched as an auxiliary payload to reduce the cost of the project. Based on these reasons, the strength and stiffness of different launcher requirements have been reviewed and the most severe requirements considered as the basis of structural calculations. This approach also gives versatility to the project and does not limit it to a specific launcher. Finally, the Arian Structure for Auxiliary Payload 5, ASAP 5, requirements are selected as the baseline of structural design. The strength and stiffness requirements applied to the structural design are indicated in Table 2 .

Table 2

Structural requirements from ASAP5 [26].

\begin{tabular}{llll}
\hline Requirement & Longitudinal & Lateral \\
\hline Strength & Acceleration $(\mathrm{g})$ & $-7.5 \mathrm{~g} /+5.5 \mathrm{~g}$ & $\pm 6 \mathrm{~g}$ \\
Stiffness & Fundamental freq. & $\geq 90 \mathrm{~Hz}$ & $\geq 45 \mathrm{~Hz}$ \\
\hline
\end{tabular}

Table 1

Satellite subsystems mass budgets.

\begin{tabular}{lcl}
\hline Subsystems & Mass budget of $m_{\text {sat }}(\%)$ & Location \\
\hline Attitude Determination and Control & 10 & Distributed on trays A, B, C, D \\
Command and Data Handling & 5 & Tray B \\
Power & 23 & Tray A \\
Telecommunication & 5 & Trays B, D \\
Thermal Control & 2 & Distributed on trays A, B, C, D \\
Structure* and Payload & 55 & "Distributed on trays A, B, C, D **Tray C \\
\hline
\end{tabular}




\subsection{Static model}

To apply the quasi-static flight limit loads imposed from the launcher (see Table 2); a simple structural model has been established for static calculations. In this model the whole satellite is considered as a cantilever beam, fixed at the end connected to the launcher adapter. It is assumed that the satellite is under an axial load associated to axial accelerations, plus a uniform load distribution in a lateral direction, associated to lateral accelerations as seen in Fig. 2(a).

According to the satellite static model, Fig. 2(b), the geometrical properties are determined by $A_{\text {sat }}=4 A_{b}+$ $2 l_{X Y} t_{s p}+2 h t_{s p}, \quad Q=A_{b} h+t_{s p} l_{X Y} h / 2+t_{s p} h^{2} / 4$ and $I_{x}=A_{b} h^{2}+$ $t_{s p} l_{X Y} h^{2} / 2+t_{s p} h^{3} / 6$, where $A_{\text {sat }}$ is the satellite model cross section area, $Q$ and $I_{x}$ are the satellite model first and second moments of area respectively, $A_{b}$ is the equal leg angles L-bar cross section area, $l_{X Y}$ is the satellite dimension in the $X Y$ direction, $h$ is the distance between two parallel side panels, $h=l_{X Y}-2 t_{s p}$, and $t_{s p}$ is the side panel thickness.

The design loads are applied based on the quasi-static flight limit loads of the launcher as indicated in Table 2, and uncertainty and safety factors are included. Thus, the longitudinal and lateral forces are calculated by $F_{\text {long/late }}=$ $m_{\text {sat }} n_{\text {long/late. }}$ SF.UF, where $F_{\text {long/late }}$ is longitudinal or lateral forces, $m_{\text {sat }}$ is the satellite total mass, $n_{\text {long/late }}$ is longitudinal or lateral load factors (launcher accelerations), $S F$ and $U F$ are design safety and uncertainty factors respectively.

According to the structural model, and from the basis of the strength of materials, the maximum normal stress which results from direct longitudinal force and lateral force bending moment is calculated by $\sigma_{\max }=M_{\max } h /$ $2 I_{x}+F_{\text {long }} / A_{\text {sat }}$, and the maximum shear stress is $\tau_{\max }=V_{\max } Q / I_{x} l_{X Y}$, where $M_{\max }$ is the maximum bending moment and $V_{\max }$ the maximum shear force.

Also, the maximum deflection of the satellite can be calculated from $\delta_{\max }=q l_{Z}^{4} / 8 \mathrm{E} I_{x}$. Where $q=F_{\text {long }} / l_{Z}$ is the lateral uniform load distribution, $l_{Z}$ is the satellite dimension in $Z$ direction, and $E$ is Young's modulus.
In static verification a buckling analysis is also carried out for each of the primary structure elements. Buckling of equal leg angles $\mathrm{L}$-bars is determined by $P_{c r}=\pi^{2} \mathrm{EI}_{x b} / L^{2}$, where $P_{c r}$ is the critical buckling load, $I_{x b}$ is the second moment of area for equal leg angles $L$-bar and $L^{\prime}$ is the effective length for equal leg angles L-bar which depends on the boundary conditions and in the worst case is twice of the L-bar length [24].

Buckling of thin plates at the satellite side panels may occur from compression, shear, bending and also under combined stress situations [22]. In a static model, for each pair of in-front side panels, two cases of buckling are assumed. First, buckling just from the compression stress, and second, buckling from combined stresses, including bending and shear. The critical buckling stress of the side panels is determined from $\sigma_{c r}=\pi^{2} k_{c} \mathrm{E} / 12\left(1-v^{2}\right)\left(t_{s p} / l_{X Y}\right)^{2}$, where $k_{c}$ is the buckling coefficient which can be obtained based on the plate dimensions and buckling source for different cases, and $v$ is Poisson's ratio. The critical buckling loads of equal leg angles L-bars, the side panel's critical buckling stress, and the applied loads, are used to determine the corresponding margin of safety of each primary structure element and to check if it is greater than zero [22].

\subsection{Dynamic model}

Being launched as an auxiliary payload can be considered as one of the constraints of low cost universityclass microsatellites. In this case there exist strict stiffness requirements imposed by the launcher. These requirements are checked by Finite Element Analysis (FEA) and testing during the detailed design phase. But at the early phases of the design, and before freezing the satellite total mass and dimensions using FEA methods for trade-off studies seem to be a slow and unnecessary process. In this work, in order to estimate the satellite's natural frequencies hereafter, the satellite structure is modeled to an equivalent four degrees of freedom (4-DOF) mass-spring system in both longitudinal and lateral directions, as seen in Fig. 3. a

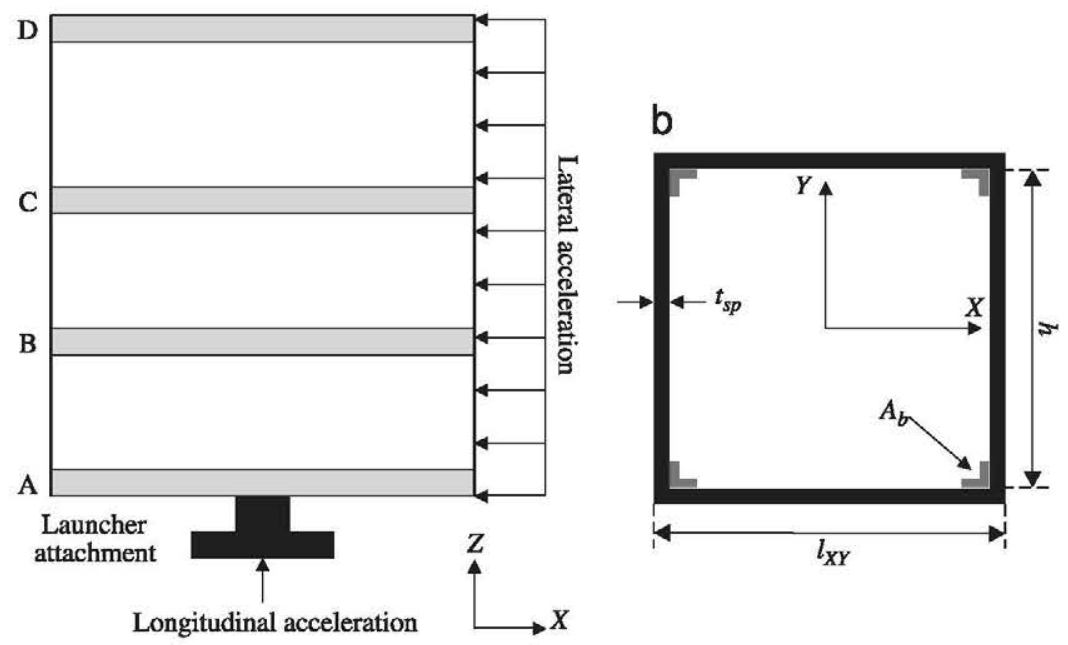

Fig. 2. Satellite static model: (a) loads imposed on the satellite (b) model cross section area. 


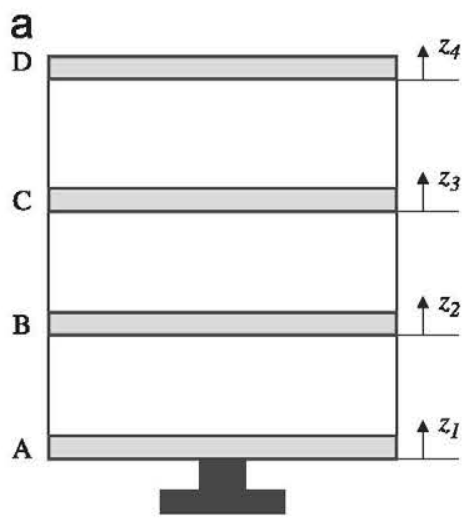

Launcher attachment b

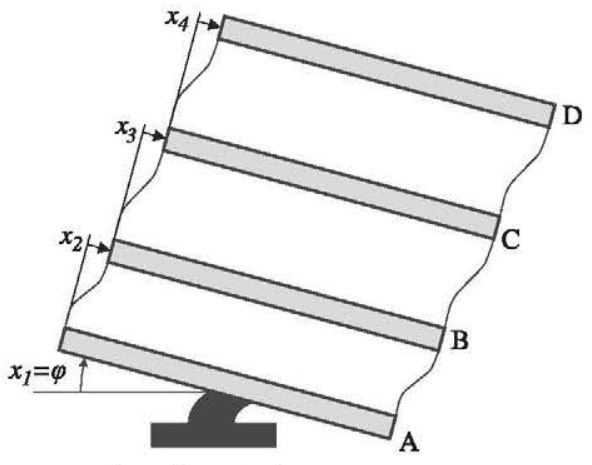

Launcher attachment

Fig. 3. Satellite dynamic model: (a) longitudinal direction (b) lateral direction.

The equivalent model is the representation of microsatellite free vibration behavior. This simplification enables rapid trade-off studies using analytical analysis with the reasonable accuracy needed for the conceptual design phase [10].

In Fig. 3(a) the free vibration response in satellite longitudinal direction ( $Z$ axis) can be described by $[\mathrm{M}]\{\ddot{Z}\}+$ $[K]\{Z\}=\{0\}$, where $\{Z\}$ is the state vector of four degrees of freedom in $Z$ direction, $[\mathrm{M}]$ is the mass matrix and $[\mathrm{K}]$ is the stiffness matrix. The mass matrix elements consist of $m_{1}, m_{2}$, $m_{3}$ and $m_{4}$, which are lumped mass considered for trays A, B, $\mathrm{C}$ and $\mathrm{D}$ respectively according to the mass distribution given in Table 1. The stiffness matrix elements consist of the equivalent longitudinal stiffness of the launcher adapter, equal leg angles L-bars and side panels between each two trays. The equivalent longitudinal stiffness of the launcher adapter is assumed to be $k_{1}=2.71 \times 10^{7}[\mathrm{~N} / \mathrm{m}]$. This stiffness is from the experience of the UPMSat-1 project [10]. The equivalent longitudinal stiffness of the satellite between each two trays can be calculated from $k_{i}=4\left(\mathrm{E} A_{b} / l_{b}+\mathrm{E} A_{s p} / l_{s p}\right)$, $\{\mathrm{i}=2,3,4\}$. Where $A_{b}$ and $A_{s p}$ are the cross section area of the equal leg angles L-bar and side panel, $l_{b}$ and $l_{s p}$ are the length of the equal leg angles L-bar and side panel between each two trays respectively.

Based on stiffness and mass matrices, the equations of motion for the equivalent 4-DOF in the longitudinal direction can be written as:

$$
\begin{aligned}
& {\left[\begin{array}{cccc}
m_{1} & 0 & 0 & 0 \\
0 & m_{2} & 0 & 0 \\
0 & 0 & m_{3} & 0 \\
0 & 0 & 0 & m_{4}
\end{array}\right]\left\{\begin{array}{l}
\ddot{z}_{1} \\
\ddot{z}_{2} \\
\ddot{z}_{3} \\
\ddot{z}_{4}
\end{array}\right\}} \\
& +\left[\begin{array}{cccc}
k_{1}+k_{2} & -k_{2} & 0 & 0 \\
-k_{2} & k_{2}+k_{3} & -k_{3} & 0 \\
0 & -k_{3} & k_{3}+k_{4} & -k_{4} \\
0 & 0 & -k_{4} & k_{4}
\end{array}\right]\left\{\begin{array}{l}
z_{1} \\
z_{2} \\
z_{3} \\
z_{4}
\end{array}\right\}=\left\{\begin{array}{l}
0 \\
0 \\
0 \\
0
\end{array}\right\}
\end{aligned}
$$

In the lateral direction the first degree of freedom is related to the rotational motion at the microsatellite and launcher attachment point. The other three degrees of freedom correspond to the lateral displacement of trays $\mathrm{B}, \mathrm{C}$ and $\mathrm{D}$.
From mass-spring equations of motion, the mass and stiffness matrices of the satellite free vibration model in the lateral direction are obtained as:

$$
\begin{aligned}
& {\left[\begin{array}{cccc}
I_{m} & 0 & 0 & 0 \\
0 & m_{2} & 0 & 0 \\
0 & 0 & m_{3} & 0 \\
0 & 0 & 0 & m_{4}
\end{array}\right]\left\{\begin{array}{l}
\ddot{\varphi} \\
\ddot{x}_{2} \\
\ddot{x}_{3} \\
\ddot{x}_{4}
\end{array}\right\}} \\
& +\left[\begin{array}{cccc}
k_{\varphi} & 0 & 0 & 0 \\
0 & k_{5}+k_{6} & -k_{6} & 0 \\
0 & -k_{6} & k_{6}+k_{7} & -k_{7} \\
0 & 0 & -k_{7} & k_{7}
\end{array}\right]\left\{\begin{array}{l}
\varphi \\
x_{2} \\
x_{3} \\
x_{4}
\end{array}\right\}=\left\{\begin{array}{l}
0 \\
0 \\
0 \\
0
\end{array}\right\}
\end{aligned}
$$

where, $I_{m}$ is the satellite moment of inertia with respect to the $X$ axis positioned in the launcher adapter plane. The equivalent rotational stiffness of the launcher adapter is considered as $k_{\varphi}=3 \times 10^{7}[\mathrm{~N} / \mathrm{m}$ ] according to [10]. The equivalent lateral stiffness of equal leg angles L-bar is determined by $k_{b j}=12 \mathrm{E} I_{x b} / l_{b}^{3},\{j=5,6,7\}$. Also, for each of two shear tolerating side panels the equivalent lateral stiffness is calculated by $k_{s p j}=F_{r} G l_{X Y} t_{s p} / l_{s p}$, where $F_{r}=0.04$ is the reduction factor because of the incomplete rigid boundary condition for side panels, and $G$ is the shear modulus. The equivalent lateral stiffness of the satellite between each two trays is the sum of the stiffness of structure elements, $k_{j}=4 k_{b j}+2 k_{s p j},\{j=5,6,7\}$. The value of $F_{r}$ is determined according to the comparison between the analytical and experimental vibration analysis of the UPMSat-1 project.

Considering the mass and stiffness matrices for the satellite model in both longitudinal and lateral directions, the natural frequencies of the satellite are obtained by solving the eigenvalue problem $\left(\mathrm{A}-\omega_{\mathrm{n}}^{2} I_{m}\right)=0$, where $\mathrm{A}=\mathrm{M}^{-1} \mathrm{~K}$ and in which $\mathrm{M}^{-1}$ is the inverse mass matrix, $\mathrm{K}$ the stiffness matrix, and $\omega_{\mathrm{n}}$ the satellite angular frequency. The satellite natural frequency is obtained by $f_{\mathrm{n}}=\omega_{\mathrm{n}} / 2 \pi$.

\section{Structure sizing tool}

In order to form a rapid sizing tool for trade-off studies, different parts of static and dynamic models are 
combined in a unified code developed in MATLAB. Different steps of structure sizing tool are seen in Fig. 4.

The hypotheses behind setting different steps are described hereafter; in step 1 a database consisting of primary structures properties and an initial value for the satellite total mass are introduced. Different primary structures properties as well as system level variables such as satellite total mass and dimensions are indicated in Table 3.

In step 2, based on criteria described in Section 3.1, a static analysis is done on each set of primary structures. Then, from different acceptable structure sets with a positive design margin of safety, the one with minimum mass is selected. According to the selected structures, the satellite total mass is calculated. If the difference between $m_{i}$, the initial mass introduced to the structure sizing tool, and $m_{c}$, the satellite total mass calculated by the structure sizing tool, is less than $0.5 \mathrm{~kg}$, a dynamic calculation starts in step 3 . If not, the differential mass $\left(m_{i}-m_{c}\right)$ is allocated

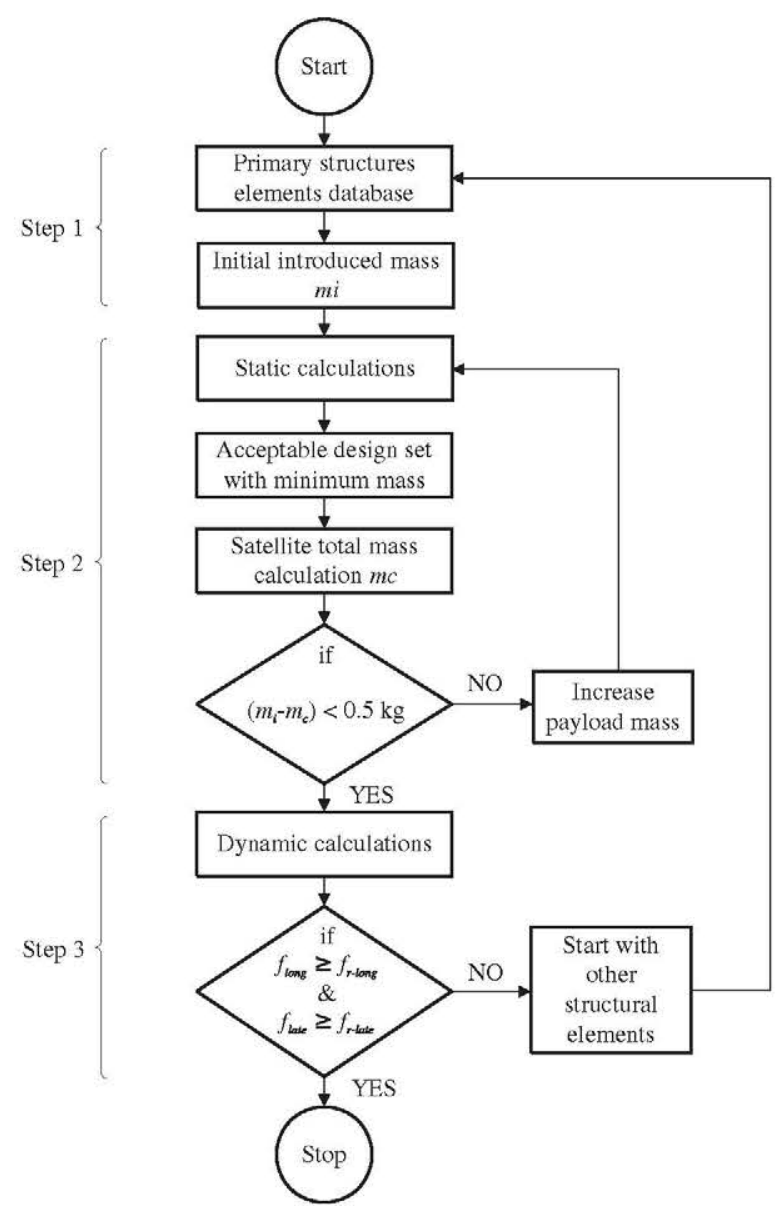

Fig. 4. Structure sizing tool calculation steps. to the payload mass budget and static calculations are repeated. In step 3 , based on the satellite dynamic model described in Section 3.2, the satellite natural frequencies in both longitudinal and lateral directions are calculated. If the natural frequencies obtained meet the launcher frequency requirements, the sizing process stops. If not, the sizing process starts from step 1 with the elimination of the current primary structure set.

It should be noted that at the beginning of the algorithm the sum of structure and payload mass budgets are defined as $55 \%$ of satellite total mass. Thus, lower structure mass results in more available mass for the payload.

\section{Case studies}

In order to see the effect of different design variables on the whole design, some specific design cases inside the design space are studied.

Design variables consist of three primary structure elements properties as well as two system level variables. The structure properties include the thickness of side panels and the thickness and leg length of equal leg angles L-bars. The system level variables are the satellite dimensions in the $X Y$ and $Z$ directions. According to the requirements of most launchers, and considering the ease of the manufacturing process, the range of practical variations of these variables are selected as seen in Table 3 .

Also, to have an evaluation medium for the design team, two design indicators are defined as: $\alpha$ which is the structure mass budget, and $\beta$ which is the payload mass budget.

As seen in Fig. 5, by increasing the satellite total mass for a fixed dimensions design, the structure mass budget

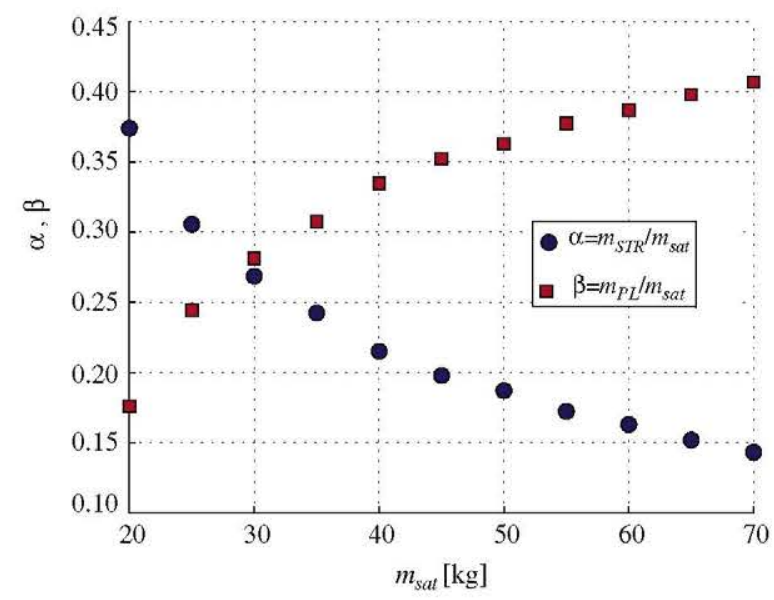

Fig. 5. Satellite total mass, $m_{\text {sat }}$, effect on structure mass budget, $\alpha$, and available mass budget for payload, $\beta$, when $l_{X Y}=0.40 \mathrm{~m}$ and $l_{Z}=0.50 \mathrm{~m}$.

Table 3

Structure sizing tool variables.

\begin{tabular}{llllll}
\hline Variable & $m_{\text {sat }}(\mathrm{kg})$ & $t_{s p} \times 10^{-3}(\mathrm{~m})$ & $d_{b} \times 10^{-3}(\mathrm{~m})$ & $t_{b} \times 10^{-3}(\mathrm{~m})$ & $l_{X Y}(\mathrm{~m})$ \\
\hline Variation limit & {$[20,70]$} & {$[1,5]$} & {$[20,30]$} & {$[1,5]$} & {$[0.40,0.50]$}
\end{tabular}



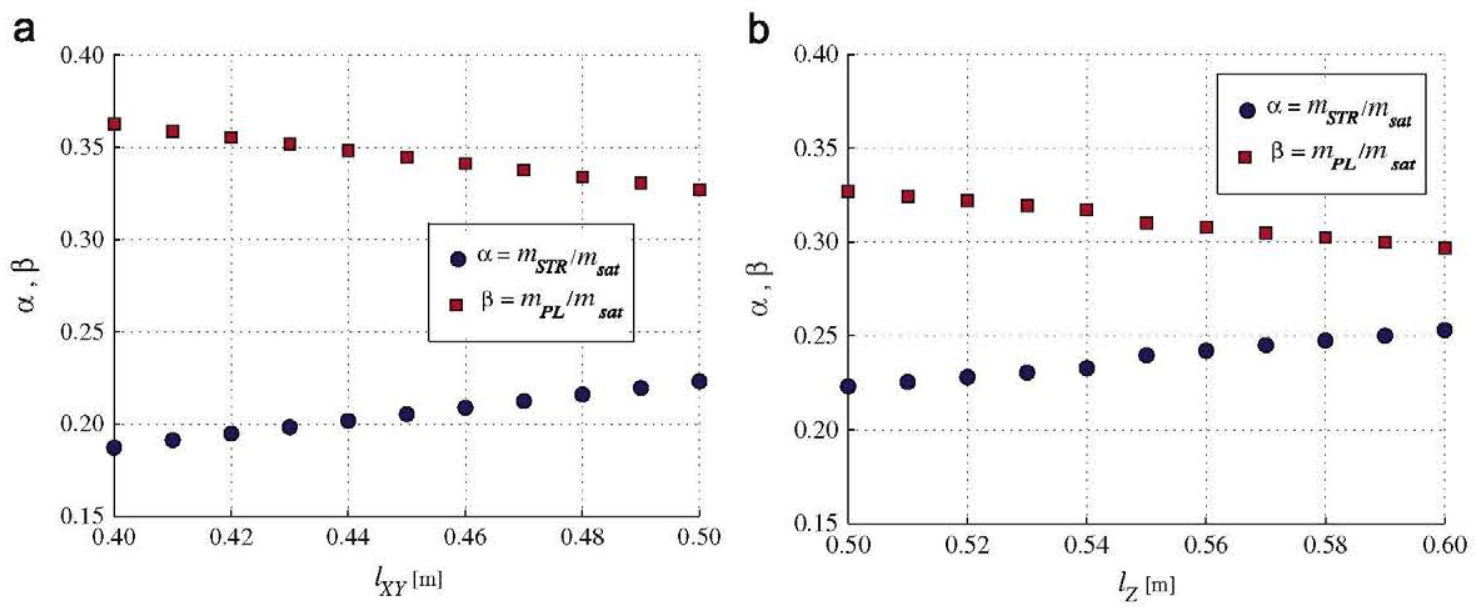

Fig. 6. Satellite dimensions effect on structure mass budget, $\alpha$, and available mass budget for payload, $\beta$, when $m_{\text {sat }}=50 \mathrm{~kg}$, (a) $l_{Z}=0.50 \mathrm{~m}$, (b) $l_{X Y}=0.50 \mathrm{~m}$.

decreases. This results in more available mass budget for the payload, which is promising when there is no exact information on the payload. On the other hand, it is always desirable to have the minimum satellite total mass in order to reduce the launch cost. Thus, two conflicting objectives have been achieved from the obtained results. The first one is the maximum available mass budget for the payload which is achieved when the structure mass budget decreases, and the second one is the minimum satellite total mass which is met when the structure mass budget increases. This result demonstrates the appropriate potential for application of the multiobjective optimization to the problem.

In Fig. $6(a, b)$, it can be seen that by increasing the dimensions of the satellite the structure mass budget increases, as expected. On the other hand, the increase of the side surface of the satellite by increasing the satellite dimension in any $X Y$ or $Z$ direction should be taken into account. This enables the power subsystem to produce more power by providing a bigger surface for mounting solar cells. This result gives a valuable insight into the interaction of the structure and power subsystems at the early phases of the design.

\section{Multiobjective optimization}

Based on the results from the structure sizing tool seen in Section 5, it can be seen that there are conflicting objectives involved in the design space. Due to this fact, a multiobjective optimization approach to the structure sizing tool is implemented. The important goal in this process is to look for the most appropriate Pareto-optimal solutions in a given design space and to select the best design baselines for future detailed design phases. The target design space for searching for the Pareto-optimal solutions is the output of the structure sizing tool for different values of variables indicated in Table 3 . Based on the satellite modeling described in Section 3, it can be seen that the design space contains discrete variables and non-linear design relationships. Thus, an appropriate optimization algorithm should be applied to the structure sizing tool.

There exists a vast body of research works related to applying optimization algorithms to engineering design problems. But optimization algorithms suitably applicable to non-continuous problems like spacecraft design are limited. This is even more limited when a multiobjective optimization is desired. The developed sizing tool can be considered as an extensive combinatorial problem. This means that applying traditional mathematical methods to this problem requires enumeration to be adjusted to the algorithm and to cover all possibilities. In this case, it is appropriate to apply non-traditional optimization based methods, such as stochastic search techniques.

\subsection{Genetic algorithm as multiobjective optimization method}

Evolutionary design optimization algorithms have been used in some works related to spacecraft design problems. In [15] Riddle Taylor, E., has demonstrated the applicability of different optimization methods, including a nontraditional heuristic method for an example spacecraft conceptual design problem. It can be concluded from his work that a heuristic method can be applied appropriately to the spacecraft design problem. In [16] Mosher T., also has showed the applicability of a GA as an evolutionary optimization method of spacecraft design. In [27] by Jones, D.F. et al., it is claimed that $70 \%$ of complex systems optimization research works used GA as the primary algorithm to solve various multidisciplinary problems. In [28] by Boudjemai, A. et al., a GA has been used for spacecraft structural design and in [29] by Ravanbakhsh, A. et al., the MATLAB GA toolbox has been applied to the conceptual design of earth observation small satellites.

Based on all the abovementioned researches, for the optimization purpose of this work a multiobjective genetic algorithm [30] is used to find the best design alternatives in the design space. 
6.2. Minimum satellite total mass versus maximum payload mass budget

As highlighted in the introduction, for university-class microsatellite projects there are uncertainties about the payload at the early design phases. Therefore, stepping through a way which provides more mass budget for the payload can be considered an important criterion of optimization in such projects. Also, minimization of the satellite total mass is always demanded to reduce the total cost of the project, especially regarding the necessary assembly and testing facilities, as well as the launch cost. On the other hand, as seen in Fig. 5, lower satellite total mass results in lower mass budget for the payload. Therefore, maximum payload mass budget and minimum satellite total mass are inherently conflicting objectives. Pareto-optimal solutions concerning these two conflicting objectives gives valuable insight into the design in order to establish the optimum design baselines, such as satellite total mass and dimensions.

For our design space the Pareto-optimal solutions are obtained as demonstrated in Fig. 7. The Pareto-optimal solutions are derived by searching the whole design space by means of the MATLAB multiobjective GA toolbox [30]. The settings are determined based on previous experience in using GA for the multidisciplinary design optimization application on the conceptual design of earth observation small satellites [29]. According to Fig. 7, for all the optimum design points, the higher the satellite total mass the lower the structure mass budget, which means more mass budget available for the satellite payload. This general behavior of optimal design points is in accordance with the results shown in Fig. 5 for a case study of a satellite configuration with fixed satellite total mass $m_{\text {sat }}=50 \mathrm{~kg}$. It should be noted that in Fig. 7 the optimization algorithm searches through the whole design space while all the design variables as well as satellite total mass are varying in their allocated boundaries indicated in Table 3 .

The other interesting result is obtained by comparing the behavior of two objectives for the satellite total mass above and below $m_{\text {sat }}=40 \mathrm{~kg}$. As seen in Fig. 7, for the satellite total mass in the range of $20-40 \mathrm{~kg}$, the structure mass budget is a greater percentage of the satellite total mass. This means that for the satellite total mass in the

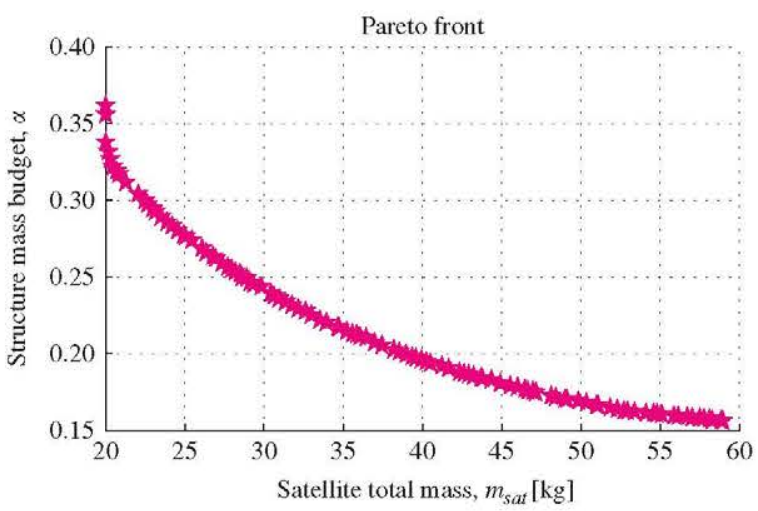

Fig. 7. Objective 1: $m_{\text {sat, }}$ satellite total mass, Objective $2: \alpha$, satellite structure mass budget. range of $20-40 \mathrm{~kg}$ the mass budget available for the payload is equal or less than the structure mass budget considering the sum of the structure and payload mass budgets is $55 \%$ of the satellite total mass. On the other hand, for the satellite total mass in the range of $40-60 \mathrm{~kg}$, the satellite structure mass budget is equal or less than $20 \%$ of the satellite total mass, which means the availability of more mass budget for the payload.

Also, in Fig. 7, considering the slope of the Pareto front, it is seen that for the satellite total mass in range of $20-40 \mathrm{~kg}$ the rate of change in structure mass budget with respect to the satellite total mass is higher compared with this rate of change for the satellite total mass in range of $40-60 \mathrm{~kg}$. For example, this can be seen considering the derivative of a polynomial of order four fitted to the Pareto front data, which is the best fitting function for the data presented in Fig.7. Actually, for satellite total mass of $40 \mathrm{~kg}$ which represents the structure mass budget of $20 \%$, the derivative of fitting function (polynomial of order four) has an inflection point. From this, we can say that when the satellite total mass is more than $40 \mathrm{~kg}$, the structure mass budget has less variation while satellite total mass is increasing. This lower rate of variation means that there exist optimum margin for obtaining as less as structure mass budget.

From this comparison it can be concluded that the selection of the satellite total mass in the range of 40$60 \mathrm{~kg}$ can be considered as an optimum approach for a university-class microsatellite project fulfilling both mentioned objectives.

\section{Conclusions}

In this work a simple and rapid approach for the structural design of low cost, university-class microsatellite projects is presented. The microsatellite configuration and structural considerations are selected in a way compatible with most launchers which provide secondary payload launch services. Furthermore, the structural elements are selected in such a way that they can be analyzed by classical structural analysis formulae. The microsatellite static and dynamic models are developed. Two design indicators, $\alpha$ which represent the satellite structure mass budget, and $\beta$ which represents the payload mass budget, are defined. The effects of structural variables, and the satellite total mass and dimensions on these design indicators are evaluated in some design cases. In order to find a Pareto-optimal inside the design space, a multiobjective GA optimization is conducted. Based on optimization results it can be concluded that selection of the satellite total mass in the range of $40-60 \mathrm{~kg}$ can be considered as an optimum approach for a universityclass microsatellite project. The result of this work is useful in the early phases of design for those projects which have no exact information about the satellite payload, unlike the classical approaches which determine the payload of the satellite.

S.I. Klimov, G.M. Tamkovich, V.N. Angarov, N.V. Elisov, Y.I. Grigoriev, O.R. Grigoryan, M.B. Dobriyan, M.N. Nozdrachev, A.P. Papkov, I.V. Pharnakeev, V.V. Radchenko, S.I. Vasiliev, 
L.M. Zelenyi, Aerospace education program realization by means of the micro-satellite, Acta Astronaut. 56 (1-2) (2005) 301-306. M.N. Sweeting, UoSAT-1: A Review of Orbital Operations and Results, J. Inst. Electron. Radio Eng. 57 (5) (1987) S184-S194. M. Swartwout, Twenty (plus) years of university-class spacecraft, a review of what was, an understanding of what is, and a look at what should be next, in: Proceedings of $20^{\text {th }}$ Annual AIAA/USU Conference on Small Satellites, USA, 2006

P. Tortora, E. Troiani, The microsatellite research program at Università di Bologna, Acta Astronaut. 56 (7) (2005) 696-704.

H.R. Goldberg, B.E. Gilchrist, The Icarus student satellite projects, Acta Astronaut. 56 (1-2) (2005) 107-114.

F. Santoni, F. Piergentili, F. Graziani, The UNISAT program: Lessons learned and achieved results, Acta Astronaut. 65 (1-2) (2009) $54-60$.

K. Thyagarajan, J.P. Gupta, P.S. Goel, K. Jayaraman, University small satellite program-ANUSAT, Acta Astronaut. 56 (1-2) (2005) 89-97.

A. Sanz-Andrés, J. Meseguer, J.M. Perales, J. Santiago-Prowald, A small platform for astrophysical research based on the UPM-SAT 1 satellite of the Universidad Politécnica de Madrid, J. Adv. Space Res. 31 (2) (2003) 375-380.

I. Perez, P. Rodriguez de Francisco, A. Sanz-Lobera, A. Sanz-Andrés, UPM: A small satellite bus for scientific, technological and educational applications, in: Proceedings of $2^{\text {nd }}$ International Symposium of Small Satellites Systems and Services, 1994.

A. Sanz-Andrés, J. Lopez Diez, P. Rodriguez de Francisco, J. Meseguer Ruiz, L. Hernández Corporales, Proyecto del satélite UPM/LB Sat, Universidad Politécnica de Madrid, (1994).

J. Sobieszczanski- Sobieski, R.T. Haftka, Review Article Multidisciplinary aerospace design optimization: survey of recent developments, Struct. Multidiscip. Optim. 14 (1) (1997) 1-23.

T. Mosher, Applicability of selected Multidisciplinary Design Optimization methods to conceptual spacecraft design, in: Proceedings of AIAA, NASA, and ISSMO, Symposium on Multidisciplinary Analysis and Optimization, 1996, pp. 664-671.

H.R. Grooms, S.F. DeBarro, S. Paydarfar, What is an Optimal Spacecraft Structure? J. Spacecr. Rockets 29 (4) (1992) 480-483.

E. Riddle, Use of optimization methods in small satellite system analysis, in: Proceedings of 12th AIAA/USU Conference on Small Satellites, 1998.
E. Riddle Taylor, Evaluation of multidisciplinary design optimization techniques as applied to spacecraft design, in: Proceedings of IEEE Aerospace Conference, 2000, pp. 371-384.

T. Mosher, Spacecraft design using a genetic algorithm optimization approach, in: Proceedings of IEEE Aerospace Conference, 1998, pp. 123-134.

W.J. Larson, J.R. Wertz, Space mission analysis and design, 3rd edition, Microcosm Press and Kluwer, USA, 1999.

P. Fortescue, J. Stark, Spacecraft systems engineering, 3rd edition, John Wiley and Sons Inc, USA, 2003.

C.D. Brown, Elements of spacecraft design, AIAA Education Series, USA, 2002.

Y.K. Chang, K.L. Hwang, S.J. Kang, SEDT (System Engineering Design Tool) development and its application to small satellite conceptual design, Acta Astronaut. 61 (7-8) (2007) 676-690.

A. Sanz-Andrés, J. Lopez Diez, C. Cuerno Rejado, Rapid method for spacecraft sizing, Proc. Inst. Mech. Eng. Part G: J. Aerosp. Eng. 209 (1995) 165-169.

E.F. Bruhn, Analysis and design of flight vehicle structures, Jacobs Publishing Inc, USA, 1973.

W.T. Thomson, M.D. Dahleh, Theory of vibration with application, Prentice Hall, USA, 1998.

T.P. Sarafin, Spacecraft structures and mechanisms, Space Technology Library, USA, 2003.

J.H. Wu, A.Q. Liu, H.L. Chen, Exact solutions for free-vibration analysis of rectangular plates using Bessel functions, J. Appl. Mech. 74 (6) (2007) 1247-1251.

ARIANESPACE, Ariane Structure for Auxiliary Payload 5 User's Manual, Approved and issued by ARIANESPACE, 2000.

D.F. Jones, S.K. Mirrazavi, M. Tamiz, Multi-objective meta-heuristics: An overview of the current state-of-the-art, Eur. J. Oper. Res. 137 (1) (2002) 1-9.

A. Boudjemai, M.H. Bouanane, L. Merad, A.M. Si Mohammad, Small satellite structural optimization using genetic algorithm approach, in: Proceedings of 3rd International conference on Recent Advances in Space Technologies, 2007, pp. 398-406.

A. Ravanbakhsh, M. Mortazavi, J. Roshanian, Multidisciplinary design optimization approach to conceptual design of a LEO earth observation microsatellite, in: Proceedings of Spaceops 2008 Conference, 2008

MATLAB Genetic Algorithm and Direct Search Toolbox, 1984-2009, Mathworks Inc 